\title{
Weather, pollution and Covid-19 spread: a time series and Wavelet reassessment
}

\section{Olivier Damette ${ }^{1}$ and Stéphane Goutte ${ }^{2}$}

\begin{abstract}
Faced with the global pandemic of Covid-19, we need to better understand the links between meteorological factors, air quality and the virus. In the vein of a recent empirical literature, we reassess the impact of weather factors like temperatures, humidity and air quality indicators on Covid-19 daily cases in China both for Wuhan and Beijing. Using a consistent number of observations (104), we compute, for the first time, correlations but also Granger causality and above all, a spectral analysis using Wavelet methods. Our results go further previous studies and reveal the complexity of the studied relationships when both time and frequency domains are taken into account. Wavelet analysis enables us to go further usual correlations analysis. Though negative humidity impact on Covid-19 cases was expected to be relatively clear regarding previous literature based on correlations, we do not find evidence of such a result. The controversial effect of warmer temperatures on the Covid-19, often difficult to identify or sometimes identified as surprisingly positive, can negatively emerge via Wavelet analysis for some periods only. This result is however clear-cut for the Hubei Province but for the Beijing one. Finally, our results reveal a bi-directional causality between air quality and the number of infected people. Short-run causality from Covid-19 to air quality (better induced air quality) via lockdown policies disappear in a medium-run and turns to become a significant causal link from induced air quality improvement to Covid-19 daily cases (reduction of infected people).
\end{abstract}

1 BETA (CNRS, INRAE), University of Lorraine, University of Strasbourg and Climate Economic Chair Paris Dauphine, France.

2 Université Paris-Saclay, UVSQ, CEMOTEV, 78280, Guyancourt, France 
- The study reassesses the link between local weather, air quality and Covid-19 epidemic in China using time series (TS) with 104 observations for China.

- Wavelet analysis enables us to go further usual correlations and TS analysis and reveal the complexity of the relationships.

- Wavelet analysis about temperature and humidity effects shows that the relationship between weather and Covid-19 is complex and not uniform regarding time and frequency.

- Humidity impact on Covid-19 epidemic seems to be not clear.

- Temperature positive effect Covid-19 epidemic turns to be negative for some periods only.

- Short-run causality from Covid-19 via lockdown disappear in a medium-run and turns to become a significant causal link from induced air quality improvement to Covid-19 daily cases.

\section{Introduction}

Faced with the global pandemic of Covid-19, declared by World Health Organization (WHO) on March 11th 2020, we need to better understand the behavior of the virus and especially its stability in different climatic environments. On a public policy point of view (sanitary, education, economic policies), it is crucial to evaluate the probability that the Covid-19 virus can decline or even disappear with Spring and Summer meteorological conditions. Though studies about survival times of the Covid-19 virus on surfaces are still under investigation, Bukhari and Jameel (2020) underlined that the spread of viruses depends upon environmental 
factors, with many respiratory pathogens showing seasonality and decreased transmission rates in warmer humid climates. According to models developed by Araujo and Naimi (2020), temperate warm and cold climates are more favorable to spread of the current Covid-19 virus. Some researchers investigated empirically if climatic factors (temperatures, humidity, wind speed, solar radiation etc...) could stop the spread of the epidemics. It is a legitime question regarding previous literature about the previous SARS virus. Chan et al. (2011), for instance, have shown that climate factors have probably impacted the outbreak of SARS - virus viability was rapidly lost at higher temperatures and higher relative humidity - and lead to different epidemics curves in countries with subtropical and tropical areas, also considering airconditioned environments. This result is in line with previous studies concerning influenza (Dalziel et al., 2018) and previous SARS coronavirus (Casanova et al., 2010, Yuan et al., 2006) regarding the relationships between climatic factors, urbanization and air quality and epidemics suggesting that cold and dry conditions increase the transmission of the virus (Baker et al., 2020).

without effective control measures, strong outbreaks are likely in more humid climates and summer weather will not substantially limit pandemic growth

However, the debate remains controversial: Wu et al. (2020) found effectively that high temperatures and high humidity should help to prevent the Covid-19 by reducing the transmission but Jamil et al. (2020) do not find evidence of an association between high temperatures (up to $20^{\circ} \mathrm{C}$ ) and the spread rate. On a public policy point of view, this question is however crucial to better manage sanitary and quarantine policies according to the dynamics of the infected people. An effect of climatic factors could help authorities to stop the epidemics as a complement to sanitary policies. Recently, Hougeveen (2020) indicated that there is a 
possibility that Covid-19 will be seasonal "going away" in May due to increasing pollen during Spring in non-tropical countries. So, what is the probability that weather changes impact the Covid-19 spread rate?

Air temperature and humidity are likely to directly impact the stability of the virus. But climatic factors are likely to impact the Covid-19 outbreak indirectly via air quality. Indeed, air quality, related or not to the meteorological conditions, is likely to impact the epidemics outbreak. Pollution is indeed a factor that is likely to perturb the immune system and thus increase the spread of infectious diseases (Caren 1981; Bauer et al. 2012) like the coronavirus family. Ogen (2020) explained that the long-term exposure to highest $\mathrm{NO}_{2}$ concentrations may be one of the most important contributors to fatality caused by the COVID-19 virus in some European regions in France, Italy, Germany and Spain. In this way, lockdown policies are useful since they can also impact indirectly the Covid-19 outbreak by improving the air quality due to the strong induced reduction of emissions. For instance, Tobias et al. (2020) outlined the net improvement of air quality due to the lockdown in Barcelona.

A very recent empirical literature has emerged on this topic to test the links between climatic factors (temperature, wind, precipitations, solar radiation), air pollution and the number of Covid-19 daily cases but the results are not completely clear. If air quality and humidity seem to negatively and significantly affect the number of infected people, results about temperatures are less clear-cut. Heterogeneity of cases, samples, countries or methods were probably one explanation.

Most of papers focused on China and suggest that the temperature variation and humidity may be important factors affecting the Covid-19 outbreak. Wu et al. (2020) found evidence, using 
cross-sectional and panel linear regressions, that high temperature and high humidity significantly reduce the transmission of Covid-19 in China. Ma et al. (2020) find a positive association between Covid-19 daily death counts and diurnal temperature range, but negative association for relative humidity. Xie and Zhu (2020) indicate that mean temperature has a positive linear relationship with the number of Covid-19 cases with a threshold of $3{ }^{\circ} \mathrm{C}$ but they do no find clear evidence supporting that case counts of Covid-19 could decline when the weather becomes warmer. Qi et al. (2020) for Mainland China, from January 20, 2020 to February 11, 2020, assess the province-specific associations between meteorological variables and the daily cases of Covid-19 and find that temperatures and relative humidity showed significantly negative associations with Covid-19 with a significant interaction between them in the Hubei province. However, these associations were not consistent throughout Mainland China.

Furthermore, studies have been recently conducted for other countries and cities beyond China. Mohsen et al. (2020) reveal that precipitations and air temperatures have no effect on the Covid19 outbreak in Iran, Coccia (2020) use data on 55 Italian province capitals and explained that the vast diffusion of Covid-19 in North of Italy is correlated with air pollution of cities measured with days exceeding the limits set for PM10 or ozone in previous years. Sahin (2020), for Turkey, conducted Spearman's correlation tests by taking into account a 14 days incubation period and showed that the highest correlations were observed for wind speed 14 days ago, and temperature on the day, respectively. Very recently, Bashir et al. (2020) outlined that average temperature, minimum temperature, and air quality were significantly associated with the Covid-19 pandemic in New York based on Kendall and Spearman rank correlation tests but they did not discuss the sign of their coefficients. Briz-Redón and Serrano-Aroca (2020) found no significant effects for Spain by incorporating control variables like non-meteorological 
factors such as population density, population by age, number of travellers and number of companies.

However, as recently outlined by Baker et al. (2020), the relative importance of climate drivers is not fully characterized; with limited data on the current epidemic, these preliminary results are inevitably inconclusive. In this paper, we reassess the relationships between local climatic conditions, air quality and Covid-19 outbreak by developing an original time series analysis coupled with a Wavelet study conducted on more than 100 time series observations. We consider two different provinces of China: Hubei as the original cluster and Beijing as a benchmark. The China case study is voluntary chosen by considering the high number of available observations - higher than previous time studies - for this country in order to conduct a robust analysis and produce meaningful results. Wavelet analysis is more powerful than some other used methodologies, especially when applied on nonlinear and non-stationary data, like Covid-19 and meteorological series. Besides, large previous literature about China can be used as a valuable benchmark.

\section{Data and methods}

\subsection{Data}

Our data set is composed by meteorological and air quality data and epidemiological data. We used daily counts of Covid-19 by downloading data from John Hopkins University Coronavirus Resource Center repository on May 5, 2020. Hence, we use a dataset covering the January 20, 2020 to May, 5, 2020 period and thus longer than previous studies.

The meteorological and air quality data include and were retrieved from the Air Quality Open Data Platform (https://aqicn.org/data-platform/covid19/). Concerning the weather variables 
Min, Max and Average values for temperatures, pressure and humidity indexes are considered. Concerning the air quality and pollution, we consider pm10, pm2.5, no2 and CO2 variables. Again, we consider min, max and average values of these variables. Indeed, extreme values like minimum temperatures might be more relevant to identify a potential link between weather and/or air quality and the spread of Covid-19 epidemics.

In addition, we only consider China case study for statistical robustness and sample size requirements. Indeed, statistical or econometric time series works are only relevant considering a sufficient number of observations for inference purposes. In our paper, we consider 104 observations (from January 20, 2020 to May, 5, 2020) that is a suitable sample to conduct a meaningful time series analysis.

\subsection{Time series and Wavelet Analysis}

In this paper, we proceed to a quantitative analysis by computing correlations and causality tests (Granger causality, see Granger, 1969) in a first step and performing a Wavelet time series analysis in a second step. Though previous quantitative works have been conducted on different countries with more or less observations, we proceed to the first time to Granger causality and, above all, to a spectral Wavelet analysis.

In contrast to standard or complex time series modelling (as the GARCH-DCC model), the wavelet coherence approach allows us to capture the co-movement between two time series in both the time and frequency domains. We adopt the wavelet coherence methodology by using the cross-wavelet transform and cross-wavelet coherence. The value of the wavelet squared coherence gives a quantity between 0 and 1, with a high value showing strong co-movement between time series and vice versa. However, unlike the standard correlation coefficient, the 
wavelet squared coherence only takes positive values. In this context, we cannot distinguish between positive and negative correlation. A solution is to use the phase difference of Terrence and Compo (1998) to provide information on positive and negative co-movements, as well as on causal relationships between time series. Black arrows on the wavelet coherence plots indicate phase. A zero phase-difference means that the time series move together. The arrows point to the right (left) when time series are in-phase (out of phase) or are positively (negatively) correlated. An upward pointing arrow means that the first time series leads the second by $\pi / 2$, whereas an arrow pointing down indicates that the second time series leads the first by $\pi / 2$. A combination of positions is generally more common.

Wavelet has been previously used in environmental sciences, climate change issues and very recently about Covid-19 issues (Iqal et al., 2020). One property which gives interesting features for Wavelet analysis, according to Gallegati (2018), is that Wavelets are particularly suitable for analyzing complex signals, especially "no stationary, have shortlived transient components, have features at different scales, or have singularities" (see also Kumar and Foufoula Georgiou 1997). The wavelet representation allows us to represent well both good time resolution at high frequencies and good frequency resolution at low frequencies. Wavelet and time series to analyze infectious diseases and weather have been previously discussed by Imai et al. (2015) for instance. Note that the Wavelet methodology enables us to consider lagged effects of weather or air quality on Covid-19 outbreak and thus now well-known 14 days Coronavirus incubation period.

\section{Results}

First, as a benchmark, we investigate the potential association between meteorological factors and the number of Covid-19 cases by computing Kendall and Spearman correlations in the way 
of most previous studies. Our results reveal a positive association between temperature and daily Covid-19 counts for both Hubei and Beijing provinces in line with previous literature. Our results give strong significant results and are consistent for the two selected provinces. We find evidence of a negative correlation between humidity and daily cases of Covid-19. Therefore, our results confirm, in the vein of of Qi et al. (2020) or Wu et al. (2020), that high levels of humidity affect negatively the spread of the virus. However, in line with most of previous papers, temperatures seem positively correlated with daily Covid-19 counts for both China areas: higher temperatures would be not a shield against the virus regarding only usual correlations.

Concerning pollution and Covid-19 relationships, our computations reveal a negative association between pollution indexes - CO2, NO2 emissions, $\mathrm{P} 10$ and $\mathrm{P} 25$ respectively - and the number of daily cases. Thus, our results seem empirically confirm that a bad air quality can aggravate the Covid-19 disease. However, as we will see, the relationship is maybe a bit more complex. In addition, correlations have some limitations: if we go further the problem of insufficient number of observations (we are working with 104 observations), correlations and regression frameworks do not take into account reverse causality problems. For example, a negative association between air quality and the number of infected people can be the consequence of the improvement of the air quality due to the lockdown policies implemented to reduce the high number of Covid-19 cases. The causality is thus not from air quality to Covid19 declared cases but in the opposite way.

At second and more interestingly, we compute Granger causality (available online) tests for all pair-wise variables (note that we transform the Covid-19 daily cases in a stationary form to perform the Granger causality tests) and find some interesting discrepancies between Wuhan 
and Beijing. Considering different indicators of air quality (CO2 emissions, PM 2.5 index), we find evidence of a causality link from air quality to Covid-19 daily cases in Wuhan. As a consequence, this result is corroborating evidence that bad air quality is likely to increase the intensity of the epidemics. However, this relationship does not hold for Beijing. This result can be related to Qi et al. (2020) that find different results concerning Wuhan and the rest of China.

In contrast, we derive a causality from Covid-19 daily cases to air quality indicators for Beijing. This result shows that the effects from the lockdown and economic downturn by drastically reducing the pollution have probably surpassed the bad direct effects of air pollution itself on the epidemics. In that way, our results also confirm and extend the previous results from Tobias et al. (2020) in Barcelona about the air quality improving due to the epidemics. More generally, our results reveal that causality between air quality and Covid-19 epidemic is potentially bidirectional, more complex that previously identified and can be of a completely different nature across different cities and situations (urbanization level, population density, economic development etc...).

Finally, our basic causality results reveal a weak causality relationship from Temperatures (average) to Covid-19 daily cases in Wuhan, epicenter of the epidemics, but not for Beijing. Nonetheless, this relation is relatively weak (only significant at $10 \%$ level) and can explain the controversial results about the temperatures-Covid-19 link in previous literature. Once again, this result suggest that relationships can vary across different local situations.

In addition, note that concerning a potential influence of humidity and atmospheric pressure on coronavirus diffusion, we do not find any significant causal relationship. It is thus impossible 
to confirm the negative association previously derived by correlation computations here and in previous literature.

At third, we next turn to a Wavelet coherency (WTC) analysis between Covid-19 daily cases to check the robustness of previous results. The powerful of this method (robust to nonstationarity, discontinuity, outliers) can be enlightening to go further previous analysis. Our results are presented hereafter via figures 1 to 4 (all results are available upon request). The horizontal axis refers to time, while the vertical axis refers to the period in days. The white line refers to the cone of influence, an edge below which wavelet power is affected due to discontinuity and hence, difficult to interpret. The black contour denotes the $5 \%$ significance level. The level of correlation is indicated by the color on the right side of the charts; the hotter the color (moving from cool (blue) to hot (yellow)) the higher the absolute correlation value. The coherency ranges from yellow (high coherency) to blue (low coherency) to measure the degree of co-movement. Thus, the yellow color represents strong co-movement whereas blue color corresponds to weak co-movements. We observe significant high degree of comovements with Covid-19 daily cases and certain variables.

We can also identify the causality and phase. We recall that arrows indicate the phase differences between Covid-19 daily cases and the weather or air quality variables. For instance, a right arrow and a left one indicates that both Covid-19 daily cases and the weather or pollution variable are in phase and out of phase respectively. Being in phase (out of phase) indicates a positive (negative) correlation. Moreover, an upper right or lower left arrow indicate that Covid19 daily cases are leading, while a lower right or upper left arrow indicate Covid-19 daily cases values are lagging. 


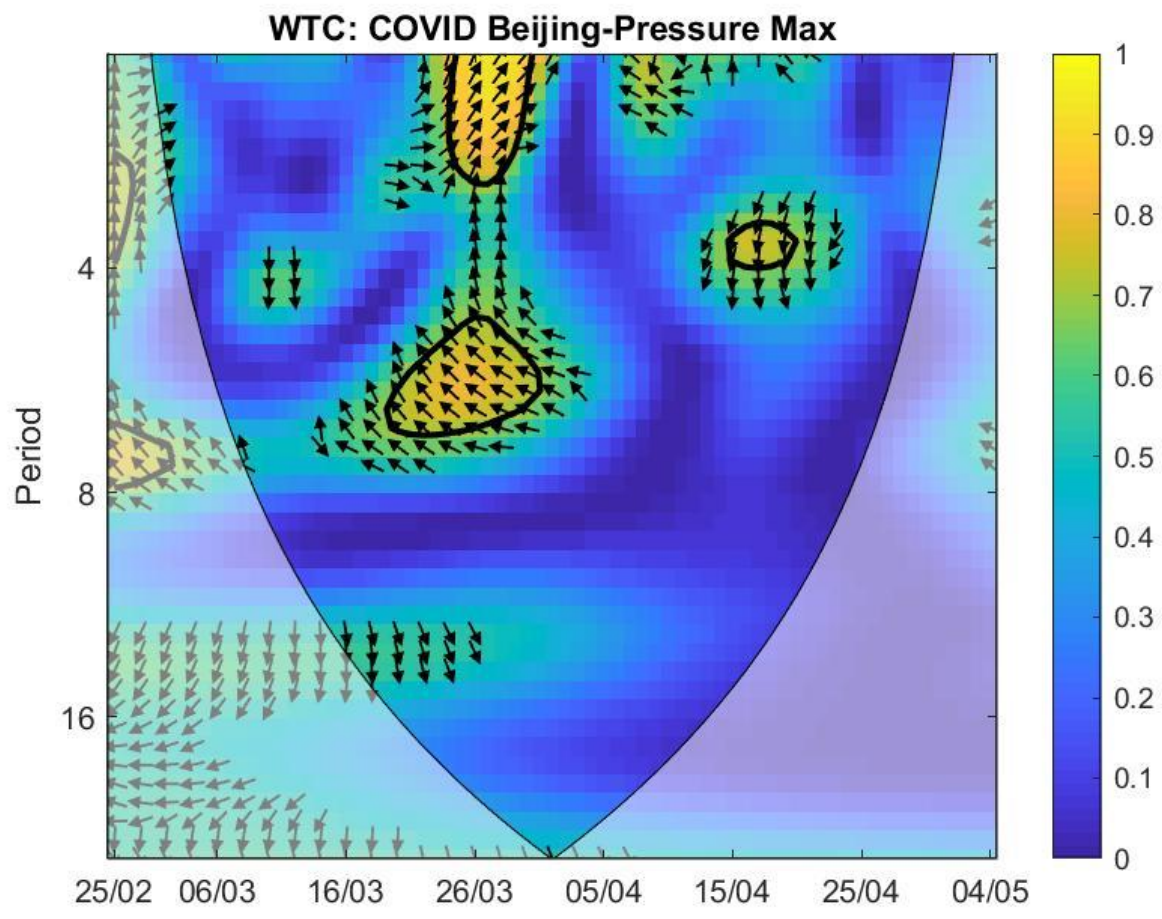

Figure 1: Wavelet coherency (WTC) between COVID-19 daily cases and Pressure in Beijing. Notes: The horizontal axis shows time, while the vertical axis refers to the period in days. The white line refers to the cone of influence, an edge below which wavelet power is affected due to discontinuity and hence, difficult to interpret. The black contour denotes the 5\\% significance level. The level of correlation is indicated by the color on the right side of the charts; the hotter the color (moving from cool (blue) to hot (yellow)) the higher the absolute correlation value 


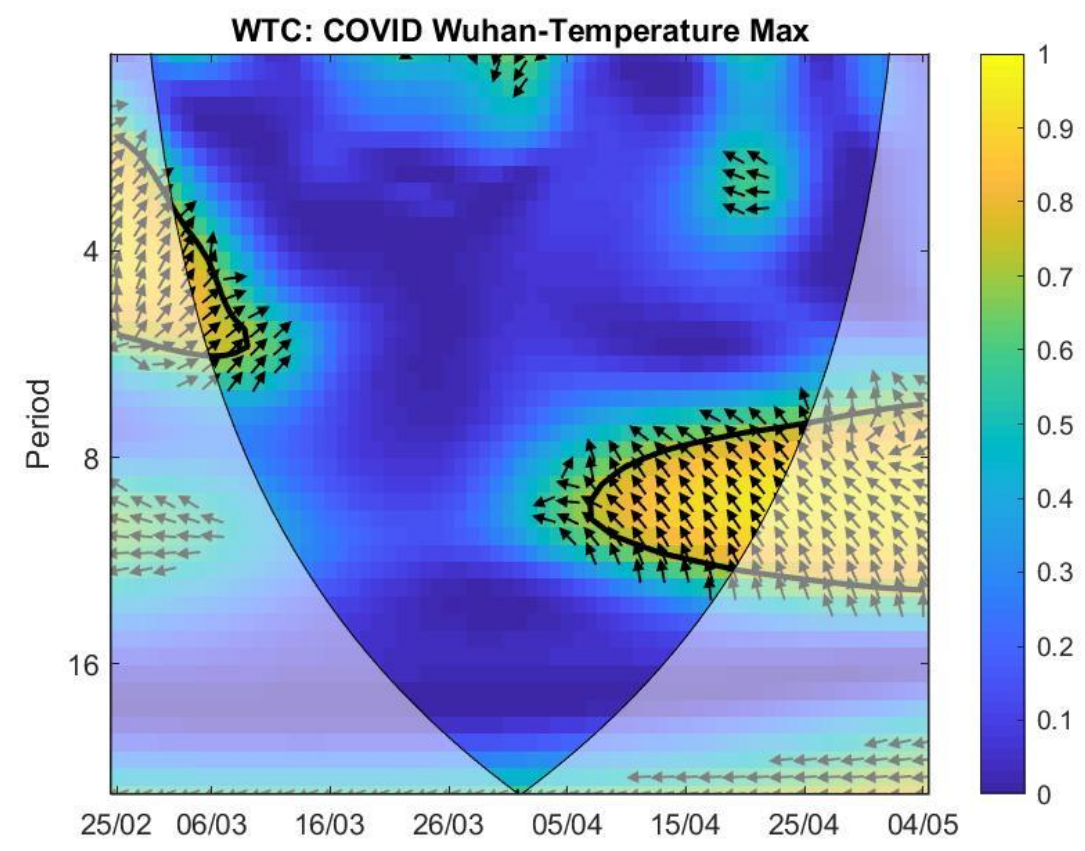

Figure 2 : Wavelet coherency (WTC) between COVID-19 daily cases and Temperature in Wuhan..

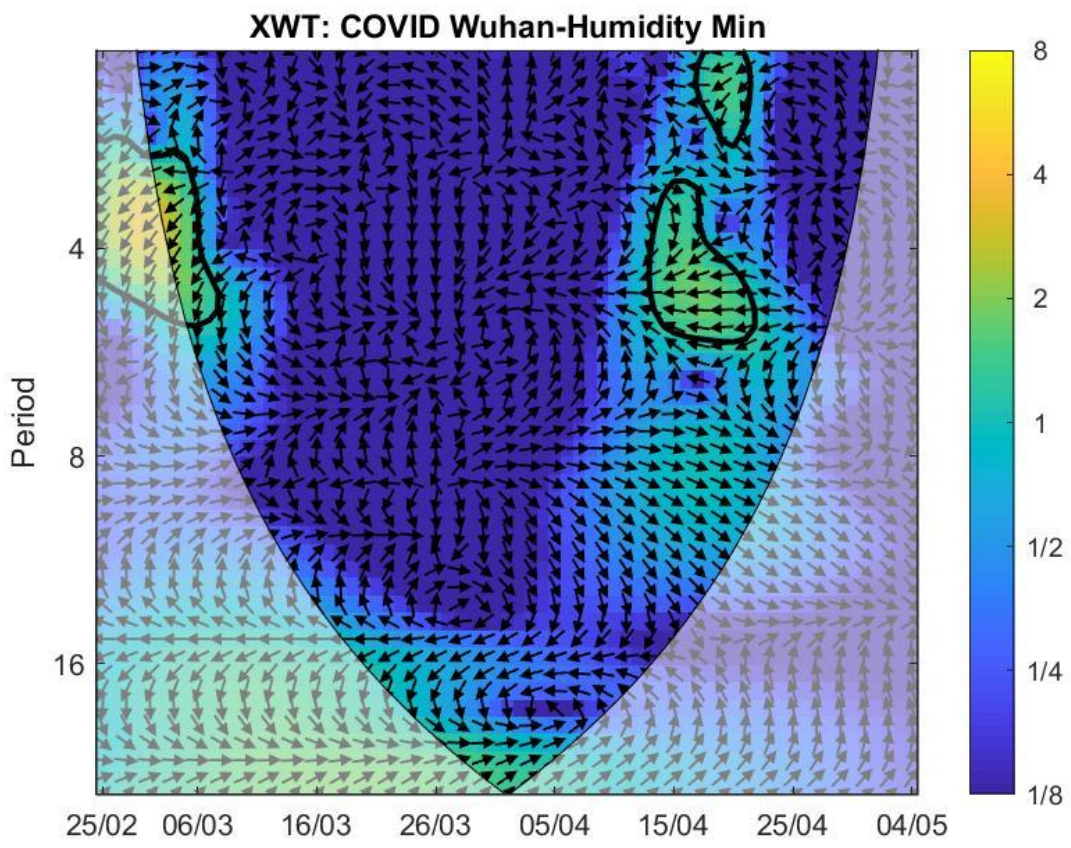

Figure 3 : Wavelet coherency (WTC) between COVID-19 daily cases and Humidity in Wuhan. 


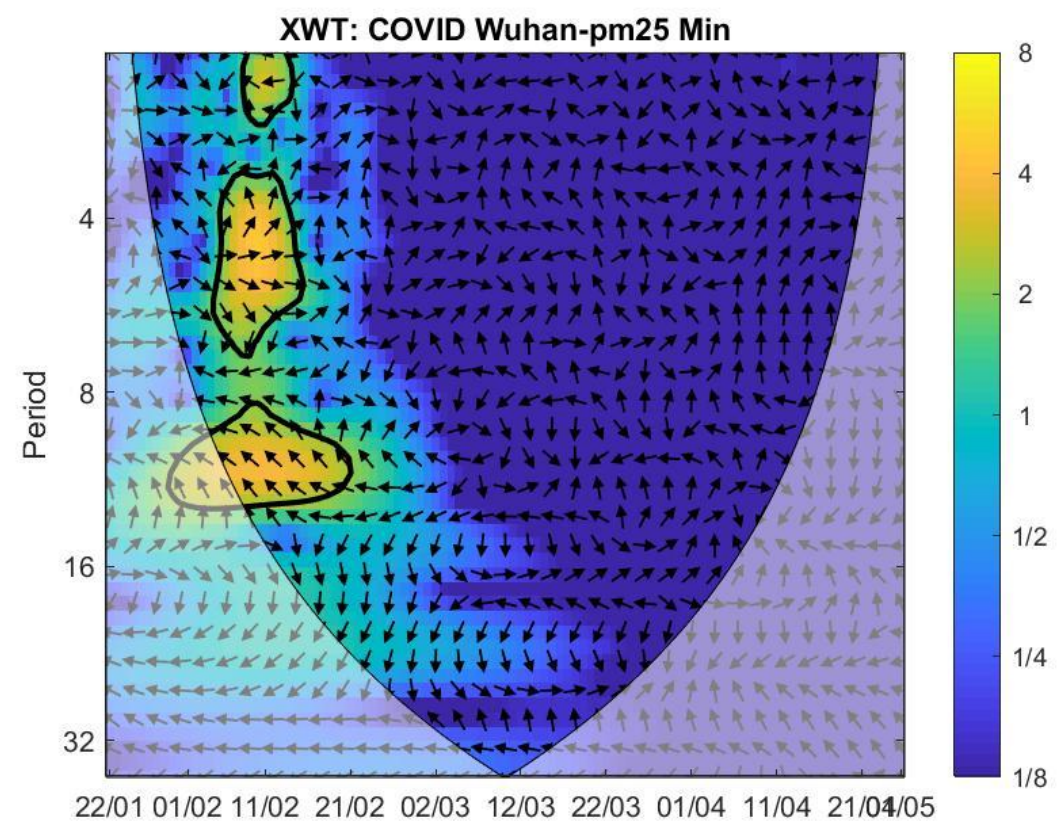

Figure 4: Wavelet coherency (WTC) between COVID-19 daily cases and Air Quality (PM 2.50) in Wuhan.

Let now present our Wavelet results. At first, we do not find any clear relationship between Pressure and Covid-19 daily cases in Wuhan whereas we derive significant co-movements for Beijing around the end of March. If we look deeper on the results, we find that for the relationship between Covid-19 cases and atmospheric pressure in Beijing (Figure 1), there is a significant period of coherence featured by co-movements in a short-run band (five days to one week) for the period of $20^{\text {th }}$ march to $2^{\text {nd }}$ April. Moreover, the arrows are in majority upper left signifying an out of phase relationship and so indicating a negative correlation between Covid19 daily cases and Pressure values with Covid-19 values lagging those of Pressure. This result is in favor of a significant Pressure (Maximum here) impact on the number of Covid-19 cases in Beijing.

At second, for the relationship between Covid-19 and Temperature in Wuhan (Figure 2), we outline a significant period of coherence with co-movements in the one to two weeks band for the period since 6th April. Moreover, the arrows are in majority upper left signifying an out of 
phase relationship and so indicating a negative correlation between Covid-19 cases and Temperature values with Covid-19 values lagging those of Temperature. In other words, the relationship from temperature to Covid-19 epidemic seems to be really negative and not positive as suggested by correlations and previous results; this result seems more in conformity with theoretical predictions. However, it is more difficult to confirm this result for Beijing. Figure 3 presents the results about Humidity for Wuhan and suggests only scarce significant co-movements. The results for Beijing (not presented here for space reasons) are not clear-cut as well. More generally, Wavelet analysis about temperature and humidity shows that the relationship between weather and Covid-19 is complex and not uniform regarding time, frequency and structural parameters across different cities and geographical areas.

Finally, concerning the relationship between Covid-19 and Air quality in Wuhan (Figure 4), we derive a significant period of coherence with co-movements in the three days to two weeks band for the period between 3rd February to $20^{\text {th }}$ February. In fact, there are two different kind of co-movements. The first one is related to a three days to one week frequency band, that is high frequency, with an in phase relationship that suggest a significant effect from the lockdown on air quality; the second one is related to a more longer band between one week and two weeks with an out of phase relationship for which the causality is working in the reverse case: better air quality is likely to reduce the number of infected people. This last result indicates a complex negative relationship between Covid-19 daily cases (and so lockdown) and Air Quality.

\section{Conclusion}


In the vein of Bukhari and Jameel (2020) and Baker et al. (2020), we share the idea that weather and especially warm and humid conditions will be probably not sufficient to curb the Covid-19 epidemics without suitable sanitary measures. Hence, higher temperatures and humidity will be not sufficient to stop the Cov-19 epidemics in Europe and North America. However, they are likely to marginally improve or deteriorate the situation, especially for vulnerable people considering variations of air quality.

For the first time, considering a robust time series dataset with 104 observations, we performed both correlation, Granger causality and spectral analysis via Wavelet methods. We find that humidity is associated with a lower number of Covid-19 daily cases regarding correlation tests. This result was expected and relatively clear in previous literature. However, our causality and Wavelet investigations are not clear on this question and do not reveal clear conclusion.

Besides, we more clarify the effect of temperatures on the virus. Though correlations coefficients between temperatures and Covid-19 cases are positive in line with some previous studies, we do not think that higher temperatures are an aggravating factor of epidemics. Indeed, Wavelet analysis reveal that if correlations coefficients are positive in average, a significant causality from temperature to Covid-19 daily cases can emerge in some periods outlining a negative causality from temperatures to the diffusion of the virus in Wuhan.

Our results also confirm that pollution and air quality, directly or indirectly, linked or not, to weather conditions, is likely to impact the coronavirus spread. At the least, it seems to have been the case in Hubei. More interestingly, our Granger causality results show that the relationship can be heterogeneous across the cities and bi-directional. The downturn induced by the epidemics and the lockdown have also been air quality-improving and can interact or 
even dominate the previous relationship from pollution to Covid-19 spread. Wavelet results suggest that both effects coexist but at different frequencies. In the short run, the dominant effect goes from the lockdown on air quality; in a longer band, between one week and two weeks, the causality is working in the reverse case and better air quality induced by lockdown policies is likely to reduce the number of infected people.

Overall, Wavelet analysis enables us to better investigate the relationships between meteorological, air quality and Covid-19 spread by distangling frequency and time periods. They reveal complex relationships and suggest to investigate multiple causality links on both time and frequency dimensions. However, as most of statistical studies, there may be several caveats to our work and we are aware that there are several other factors that may play roles in the number of affected cases. Epidemiological factors (population mobility, immunity etc...), individual health factors and personal preferences (hygiene habits for example) and structural factors (urbanization and population density, public health infrastructures and policies, political and social preferences) should be incorporated in future works when large panel data sets will be available.

Further studies are expected to confirm or not our preliminary results and above all, improve our understanding about the links between weather, pollution and epidemics. Cross-country and long run studies at a world scale will be necessary to further investigate the climate - beyond meteorological - impact on coronavirus and help to anticipate the emergence of other possible pandemics in the future. Anyway, climate change and ecology transition issues are probably crucial topics to take into account in the epidemic's analysis.

\section{References}


Araujo M.B., Naimi B. (2020). Spread of SARS-CoV-2 Coronavirus likely to be constrained by climate, doi: https://doi.org/10.1101/2020.03.12.20034728

Bashir M.F. et al. (2020). Correlation between climate indicators and COVID-19 pandemic in New York, USA. Science of the Total Environment, 728, 138835.

Bauer R., Diaz-Sanchez D., Jaspers D. (2012). Effects of air pollutants on innate immunity: The role of toll-like receptors and nucleotide-binding oligomerization domain-like receptors. The Journal of Allergy and Clinical Immunology, 129, 14-24.

Baker R.E., Yang W., Vecchi G.A., Metcalf C.J.E., Grenfell B. T. (2020), "Susceptible supply limits the role of climate in the early SARS-CoV-2 pandemic", Science, 10.1126/science.abc2535 (2020).

Briz-Redón A., Serrano-Aroca A. (2020), “A spatio-temporal analysis for exploring the effect of temperature on COVID-19 early evolution in Spain", Science of Total Environment, 728, 1 728August 2020, 138811.

Bukhari Q., Jameel Y. (2020). Will coronavirus pandemic diminish by summer? SSRN working paper 3556998 .

Caren L. (1981). Environmental pollutants: effects on the immune system and resistance to infectious disease. Bioscience, 31, 582-586.

Casanova, L. M., Jeon, S., Rutala, W. A., Weber, D. J., \& Sobsey, M. D. (2010). Effects of air temperature and relative humidity on coronavirus survival on surfaces. Appl. Environ. Microbiol., 76, 9, 2712-2717. 
Chan, K., Peiris, J., Lam, S., Poon, L., Yuen, K., \& Seto, W. (2011). The effects of temperature and relative humidity on the viability of the SARS coronavirus. Advances in virology, 2011.

Chen, H., Guo, J., Wang, C., Luo, F., Yu, X., Zhang, W., . . . Gong, Q. (2020). Clinical characteristics and intrauterine vertical transmission potential of COVID-19 infection in nine pregnant women: a retrospective review of medical records. The Lancet, 395(10226), 809-815.

Coccia M. (2020). Factors determining the diffusion of COVID-19 and suggested strategy to prevent future accelerated viral infectivity similar to COVID. Science of the Total Environment $729,138474$.

Dalziel, B. D., Kissler, S., Gog, J. R., Viboud, C., Bjornstad, O. N., Metcalf, C. J. E., \& Grenfell, B. T. (2018). Urbanization and humidity shape the intensity of influenza epidemics in U.S. cities. Science, 362(6410), 75-79.

Gallegati M. (2018). A systematic wavelet-based exploratory analysis of climatic variables. Climatic Change, 148, issue 1-2, pp. 325-338.

Granger C. W. J. (1969). Investigating Causal Relations by Econometric Models and Crossspectral Methods. Econometrica, 37, 3, 424-438.

Hougeven M. J. (2020). Pollen likely seasonal factor in inhibiting flu-like epidemics. A Dutch study into the inverse relation between pollen counts, hay fever and flu-like incidence 20162019. Science of the Total Environment, 727, 138543.

Imai C., Armstrong B., Chalabi Z., Pangtani P., Hashizume M. (2015), “Time series regression model for infectious disease and weather", Environmental Research, 142, pp. 319-327. 
Iqbal N., Zeeshan Fareed, Farrukh Shahzad, Xin He, Umer Shahzad, Ma Lina (2020). "Nexus between COVID-19, temperature and exchange rate in Wuhan City: New findings from Partial and Multiple Wavelet Coherence. Science of the Total Environment, 729, 138916.

Jamil, T., Alam, I., Gojobori, T., Duarte, C. M. (2020). No Evidence for TemperatureDependence of the COVID-19 Epidemic. medRxiv preprint doi: https://doi.org/10.1101/2020.03.29.20046706.

Lamarre, A., \& Talbot, P. J. (1989). Effect of $\mathrm{pH}$ and temperature on the infectivity of human coronavirus 229E. Canadian journal of microbiology, 35, 10, 972-974.

Ma, Y., Zhao, Y., Liu, J., He, X., Wang, B., Fu, S., . . Luo, B. (2020). Effects of temperature variation and humidity on the death of COVID-19 in Wuhan, China. Science of The Total Environment, 724, 138226.

Mohsen Ahmadi, Abbas Sharifi, Shadi Dorosti, Saeid Jafarzadeh Ghoushchi, Negar Ghanbari (2020). Investigation of effective climatology parameters on COVID-19 outbreak in Iran. Science of the Total Environment, 729, 738205.

Ogen Y. (2020), “Assessing nitrogen dioxide $\left(\mathrm{NO}_{2}\right)$ levels as a contributing factor to coronavirus (COVID-19) fatality", Science of The Total Environment, Vol. 726, 15 July 2020, 138605.

Oliveiros, B., Caramelo, L., Ferreira, N. C., \& Caramelo, F. (2020). Role of temperature and humidity in the modulation of the doubling time of COVID-19 cases. medRxiv.

Qi H., Xiao S., ... (2020). COVID-19 transmission in Mainland China is associated with temperature and humidity: A time-series analysis. Science of the Total Environment 728, 138778. 
Sajadi, M. M., Habibzadeh, P., Vintzileos, A., Shokouhi, S., Miralles-Wilhelm, F., \& Amoroso, A. (2020). Temperature and latitude analysis to predict potential spread and seasonality for COVID-19. Available at SSRN 3550308.

Şahin Mehmet (2020). Impact of weather on COVID-19 pandemic in Turkey, Science of the Total Environment 728, 138810.

Torrence, C Compo, G.P (1998). A practical guide to wavelet analysis, Bull. Amer. Meteorol. Soc. 79 pp. $61-78$.

Tosepu, R., Gunawan, J., Effendy, D. S., Lestari, H., Bahar, H., \& Asfian, P. (2020). Correlation between weather and Covid-19 pandemic in Jakarta, Indonesia. Science of The Total Environment, 725, 138436.

Wang, M., Jiang, A., Gong, L., Luo, L., Guo, W., Li, C., . . Z Zeng, J. (2020). Temperature significant change COVID-19 Transmission in 429 cities. medRxiv.

Wang J., Tang K., Feng K., Lv W. (2020). High Temperature and High Humidity Reduce the Transmission of COVID-19. SSRN Working Paper.

Xie J. Zhu Y (2020). Association between ambient temperature and COVID-19 infection in 122 cities from China, Science of the Total Environment, 724, 138201

Zhu, N., Zhang, D., Wang, W., Li, X., Yang, B., Song, J., . . Lu, R. (2020). A novel coronavirus from patients with pneumonia in China, 2019. New England Journal of Medicine. 